\title{
Mid-infrared interferometry of the Mira variable RR Sco with the VLTI MIDI instrument ${ }^{\star}$
}

\author{
K. Ohnaka ${ }^{1}$, J. Bergeat ${ }^{2}$, T. Driebe ${ }^{1}$, U. Graser ${ }^{3}$, K.-H. Hofmann ${ }^{1}$, R. Köhler ${ }^{3}$, Ch. Leinert ${ }^{3}$, \\ B. Lopez ${ }^{4}$, F. Malbet ${ }^{5}$, S. Morel ${ }^{6}$, F. Paresce ${ }^{7}$, G. Perrin ${ }^{8}$, Th. Preibisch ${ }^{1}$, A. Richichi ${ }^{7}$, \\ D. Schertl ${ }^{1}$, M. Schöller ${ }^{6}, \mathrm{H} . \mathrm{Sol}^{8}$, G. Weigelt ${ }^{1}$, and M. Wittkowski ${ }^{7}$
}

${ }^{1}$ Max-Planck-Institut für Radioastronomie, Auf dem Hügel 69, 53121 Bonn, Germany e-mail: kohnaka@mpifr-bonn.mpg.de

2 Observatoire de Lyon, 9 avenue Charles André, 69561 St.-Genis-Laval Cedex, France

${ }^{3}$ Max-Planck-Institut für Astronomie, Königstuhl 17, 69117 Heidelberg, Germany

4 Observatoire de la Côte d'Azur, Département Fresnel, UMR 6528 CNRS, BP 4229, 06304 Nice Cedex 4, France

${ }^{5}$ Laboratoire d'Astrophysique, Observatoire de Grenoble, 38041 Grenoble Cedex 9, France

${ }^{6}$ European Southern Observatory, Casilla 19001, Santiago 19, Chile

7 European Southern Observatory, Karl-Schwarzschild-Str. 2, 85748 Garching, Germany

8 Observatoire de Paris-Meudon, 5 place Jules Janssen, 92190 Meudon, France

Received 7 April 2004 / Accepted 8 September 2004

\begin{abstract}
We present the results of the first mid-infrared interferometric observations of the Mira variable RR Sco with the MID-infrared Interferometer (MIDI) coupled to the European Southern Observatory's (ESO) Very Large Telescope Interferometer (VLTI), together with $K$-band observations using VLTI VINCI. The observations were carried out in June 2003, when the variability phase of the object was 0.6, using two unit telescopes (UT1 and UT3), as part of the Science Demonstration Time (SDT) program of the instrument. Projected baseline lengths ranged from 73 to $102 \mathrm{~m}$, and a spectral resolution of 30 was employed in the observations, which enabled us to obtain the wavelength dependence of the visibility in the region between 8 and $13 \mu \mathrm{m}$. The uniform-disk diameter was found to be 18 mas between 8 and $10 \mu \mathrm{m}$, while it gradually increases at wavelengths longer than $10 \mu \mathrm{m}$ to reach 24 mas at $13 \mu \mathrm{m}$. The uniform-disk diameter between 8 and $13 \mu \mathrm{m}$ is significantly larger than the $K$-band uniform-disk diameter of $10.2 \pm 0.5$ mas measured using VLTI VINCI with projected baseline lengths of 15-16 m, three weeks after the MIDI observations. Our model calculations show that optically thick emission from a warm molecular envelope consisting of $\mathrm{H}_{2} \mathrm{O}$ and $\mathrm{SiO}$ can cause the apparent mid-infrared diameter to be much larger than the continuum diameter. We find that the warm molecular envelope model extending to $\sim 2.3 R_{\star}$ with a temperature of $\sim 1400 \mathrm{~K}$ and column densities of $\mathrm{H}_{2} \mathrm{O}$ and $\mathrm{SiO}$ of $3 \times 10^{21} \mathrm{~cm}^{-2}$ and $1 \times 10^{20} \mathrm{~cm}^{-2}$, respectively, can reproduce the observed uniform-disk diameters between 8 and $10 \mu \mathrm{m}$. The observed increase of the uniform-disk diameter longward of $10 \mu \mathrm{m}$ can be explained by an optically thin dust shell consisting of silicate and corundum grains. The inner radius of the optically thin dust shell is derived to be 7-8 $R_{\star}$ with a temperature of $\sim 700 \mathrm{~K}$, and the optical depth at $10 \mu \mathrm{m}$ is found to be $\sim 0.025$.
\end{abstract}

Key words. infrared: stars - techniques: interferometric - stars: circumstellar matter - stars: AGB and post-AGB stars: individual: RR Sco - stars: mass-loss

\section{Introduction}

Intermediate- and low-mass stars experience slow but massive mass loss while moving along the asymptotic giant branch (AGB) during the late stage of their evolution. Since nuclear processed material is dredged up to the surface and finally returned to the interstellar space via mass loss, AGB stars are believed to play an important role in the chemical evolution of the Galaxy. Mira-type AGB stars are of particular interest, because they exhibit complicated physical and chemical processes such as large-amplitude stellar pulsation, shock waves,

* Based on observations made with the Very Large Telescope Interferometer of the European Southern Observatory. grain formation, and momentum transfer from photons to dust grains.

However, the mass loss mechanism in AGB stars is not yet fully understood. In particular, the understanding of the region where material is accelerated is meager. In order to better understand the mass loss phenomenon in Mira-type AGB stars, it is crucial to obtain a comprehensive picture of the region where mass outflow is expected to be initiated, that is, the region between the top of the photosphere and the inner edge of the expanding dust shell. Recent spectroscopic observations of AGB stars with the Infrared Space Observatory (ISO) have revealed the existence of a quasi-static, warm, dense molecular envelope close to the star (e.g., Tsuji et al. 1997; 
Yamamura et al. 1999; Cami et al. 2000; Matsuura et al. 2002). It cannot be explained by non-gray hydrostatic photospheric models and has physical properties distinct from the cold, expanding circumstellar dust shell. The analyses of infrared molecular absorption/emission features such as $\mathrm{CO}, \mathrm{H}_{2} \mathrm{O}$, $\mathrm{SiO}, \mathrm{CO}_{2}$, and $\mathrm{SO}_{2}$ observed with the ISO Short Wavelength Spectrometer (SWS) suggest that the warm molecular envelope has temperatures of 1000-2000 K, hydrogen molecule number densities of up to $10^{11} \mathrm{~cm}^{-3}$, and a geometrical extension of a few stellar radii. Although this warm molecular envelope is most likely to play an important role in mass loss, its formation mechanism is not yet understood.

Mid-infrared interferometry provides a unique opportunity to probe the circumstellar environment of Mira variables. Since a large fraction of mid-infrared photons originate in regions cooler than the photosphere, mid-infrared interferometry is well suited for studying the outer atmosphere and the circumstellar dust shell, where complicated, mutually coupled physical and chemical processes take place, finally leading to the onset of mass outflows. However, up to now, only a handful of interferometric experiments in the thermal infrared have been performed, because of the difficulty in calibrating visibilities. McCarthy \& Low (1975) carried out visibility measurements at $5 \mu \mathrm{m}$, using a mask with two circular holes mounted on single-dish telescopes. McCarthy et al. $(1977,1978)$ extended this technique to the $N$ band and observed AGB stars as well as M supergiants. Gay \& Mékarnia (1988) and Mékarnia \& Gay (1990) carried out visibility measurements of the M supergiant $\alpha$ Ori at $10 \mu \mathrm{m}$ using a two-telescope interferometer. Recently, Mennesson et al. (2002) have measured $L^{\prime}$-band visibilities with the Infrared Optical Telescope Array (IOTA). The main interferometer in operation in the $10 \mu \mathrm{m}$ region at the moment is the Infrared Spatial Interferometer (ISI), which utilizes heterodyne detection using a $\mathrm{CO}_{2}$ laser local oscillator and is operated between 9.5 and $11.5 \mu \mathrm{m}$ (e.g., Townes et al. 1998; Hale et al. 2000).

The advent of MIDI (Leinert et al. 2003) at VLTI is expected to allow new possibilities, particularly with the capability to obtain spectrally dispersed interferograms over the wavelength range between 8 and $13 \mu \mathrm{m}$. While ISI operates at one fixed wavelength at a time with a spectral resolution of $\sim 5000$, MIDI can simultaneously record spectrally dispersed interferograms over the whole range of the $N$ band with a spectral resolution of $\sim 30$ or $\sim 230$.

In this paper, we present the first spectrally dispersed $N$-band interferometric observations of the Mira variable RR Sco with VLTI MIDI and, in addition, $K$-broadband observations with VLTI VINCI. RR Sco is a Mira variable with a spectral type of M6II-IIIe-M9 and a period of 281.4 days (Kholopov et al. 1988). The $V$ magnitude varies from $\sim 6$ at maximum light to $\sim 12$ at minimum light, while the lightcurve in the $K$ band obtained by Whitelock et al. (2000) shows that the $K$ magnitude varies from $\sim-0.5$ at maximum light to $\sim 0.0$ at minimum light. The IRAS $12 \mu \mathrm{m}$ flux of RR Sco is $188.7 \mathrm{Jy}$, and the distance to RR Sco is estimated to be $320 \pm 120 \mathrm{pc}$ based on the revised HIPPARCOS parallax of $3.10 \pm 1.16$ mas (Knapp et al. 2003). While oxygen-rich Mira variables often exhibit prominent emission due to silicate dust centered
Table 1. Summary of MIDI SDT observations of RR Sco: date, modified Julian Date (MJD), time of observation (Universal Time = UTC), projected baseline length $B_{\mathrm{p}}$, and position angle of the projected baseline on the sky (PA).

\begin{tabular}{rcccrr}
\hline \hline$\#$ & Date & MJD & $t_{\text {obs }}[\mathrm{UTC}]$ & $B_{\mathrm{p}}[\mathrm{m}]$ & PA [ $\left.{ }^{\circ}\right]$ \\
\hline 1 & 2003 Jun. 14 & 52805.043 & $01: 02: 21$ & 101.3 & 6.8 \\
2 & 2003 Jun. 14 & 52805.089 & $02: 09: 22$ & 101.9 & 17.2 \\
3 & 2003 Jun. 14 & 52805.213 & $05: 07: 04$ & 99.9 & 38.6 \\
4 & 2003 Jun. 14 & 52805.306 & $07: 21: 17$ & 86.1 & 47.1 \\
5 & 2003 Jun. 15 & 52806.329 & $07: 53: 18$ & 80.2 & 48.0 \\
6 & 2003 Jun. 15 & 52806.332 & $07: 58: 31$ & 79.5 & 48.0 \\
7 & 2003 Jun. 16 & 52807.350 & $08: 24: 01$ & 73.7 & 48.0 \\
\hline
\end{tabular}

around $9.8 \mu \mathrm{m}$, the IRAS Low Resolution Spectrum (LRS) of RR Sco shows very little dust feature between 8 and $23 \mu \mathrm{m}$, which allows us to better study the warm molecular envelope through the dust shell.

\section{Observations and data reduction}

RR Sco was observed with MIDI at variability phase 0.6 on three consecutive nights in mid-June 2003 within the framework of the Science Demonstration Time (SDT) program. Interferometric data were taken with a beam combiner, which produces two interferometric signals of opposite sign. A prism with a spectral resolution of $\lambda / \Delta \lambda \simeq 30$ was used to obtain spectrally dispersed fringes. The fringes were scanned by applying rapid internal optical path difference (OPD) changes of a few wavelengths around the point of zero OPD. Photometric calibration data were recorded by blocking each of the two beams alternately, immediately after recording the interferometric data. Chopping was applied in recording the photometric data to obtain sky frames (chopping parameters: $\sim 0.25 \mathrm{~s}$ on the target, corresponding to $\sim 12$ target frames, followed by $\sim 0.25$ s on the sky; this sequence was repeated $\sim 75-110$ times). No chopping was performed during the recording of the interferometric data. A more detailed description of the observing strategy is given in Leinert et al. (2004).

In total, seven observations were carried out using the $102 \mathrm{~m}$ baseline between the telescopes UT1 and UT3. Due to projection effects, the projected baseline lengths range between 74 and $102 \mathrm{~m}$, as can be seen in Table 1, where details of the individual observing runs are summarized. Calibrator stars whose angular diameter is known were observed for the calibration of the raw visibilities, and Table 2 gives an overview of these calibration stars, their uniform-disk diameters, and the MIDI SDT observations that we used for the calibration of the raw visibilities of RR Sco.

For data reduction, we use the MIDI software package based on the power spectrum analysis (Leinert et al. 2004). The first step of the reduction procedure is to read the photometric calibration data sets, to average the target and sky frames, and to subtract the average sky frame from the average target frame. The sky-subtracted target frame yields the object spectrum. This procedure is applied to both photometric data sets. Each individual interferometric frame, 
Table 2. List of calibrators used for the calibration of the RR Sco data together with their limb-darkened $\left(d_{\mathrm{LD}}\right)$ and uniform-disk diameters $\left(d_{\mathrm{UD}}\right)$, and the date and time stamp $\left(t_{\mathrm{obs}}\right)$ of their SDT observation with MIDI. For the conversion from limb-darkened diameters to uniformdisk diameters we adopted the scheme described in Wittkowski et al. (2001). The limb-darkened diameters were taken from Bordé et al. (2002), Cohen et al. (1999), and Stecklum (2004). The data set marked with a dagger was not used for deriving visibilities due to poor quality of the interferograms, but used only for extracting the spectrum.

\begin{tabular}{|c|c|c|c|c|}
\hline $\begin{array}{l}\text { Calibrator } \\
\text { HD number }\end{array}$ & $d_{\mathrm{LD}}$ [mas] & $d_{\mathrm{UD}}$ [mas] & $\begin{array}{c}\text { night } \\
(2003)\end{array}$ & $t_{\mathrm{obs}}[\mathrm{UTC}]$ \\
\hline 89388 & $5.23 \pm 0.06$ & $5.18 \pm 0.06$ & Jun. 16 & $23: 27: 39$ \\
\hline \multirow[t]{2}{*}{10380} & $2.92 \pm 0.03$ & $2.89 \pm 0.03$ & Jun. 14 & 09:37:19 \\
\hline & & & Jun. 15 & $09: 34: 22$ \\
\hline \multirow[t]{2}{*}{139063} & $4.27 \pm 0.05$ & $4.23 \pm 0.05$ & Jun. 16 & $02: 51: 58$ \\
\hline & & & Jun. 16 & 04:09:17 \\
\hline 139997 & $3.71 \pm 0.10$ & $3.67 \pm 0.01$ & Jun. 16 & $05: 31: 17$ \\
\hline \multirow[t]{2}{*}{151680} & $5.99 \pm 0.06$ & $5.93 \pm 0.06$ & Jun. 14 & $00: 32: 49$ \\
\hline & & & Jun. 14 & $05: 29: 30^{\dagger}$ \\
\hline \multirow[t]{2}{*}{152161} & $4.83 \pm 0.50$ & $4.78 \pm 0.50$ & Jun. 15 & $05: 31: 37$ \\
\hline & & & Jun. 16 & $23: 27: 39$ \\
\hline 152786 & $7.21 \pm 0.21$ & $7.14 \pm 0.21$ & Jun. 15 & 01:06:42 \\
\hline \multirow[t]{3}{*}{165135} & $3.38 \pm 0.16$ & $3.35 \pm 0.16$ & Jun. 14 & 09:03:35 \\
\hline & & & Jun. 15 & $00: 36: 11$ \\
\hline & & & Jun. 15 & 02:46:05 \\
\hline \multirow[t]{4}{*}{167618} & $11.33 \pm 0.04$ & $11.22 \pm 0.04$ & Jun. 14 & $01: 27: 39$ \\
\hline & & & Jun. 14 & $07: 57: 22$ \\
\hline & & & Jun. 15 & $07: 22: 23$ \\
\hline & & & Jun. 15 & 07:28:00 \\
\hline \multirow[t]{4}{*}{168454} & $5.78 \pm 0.15$ & $5.72 \pm 0.15$ & Jun. 14 & $02: 44: 43$ \\
\hline & & & Jun. 14 & 04:00:09 \\
\hline & & & Jun. 14 & $06: 10: 48$ \\
\hline & & & Jun. 16 & 06:35:05 \\
\hline \multirow[t]{2}{*}{187642} & $3.31 \pm 0.11$ & $3.28 \pm 0.11$ & Jun. 15 & $03: 53: 11$ \\
\hline & & & Jun. 15 & $06: 44: 35$ \\
\hline
\end{tabular}

corresponding to one individual OPD within a scan, is projected to a one-dimensional spectrum by integrating over the direction perpendicular to the spectral dispersion. Then, these one-dimensional spectra, which correspond to different OPDs, are put together in the direction perpendicular to that of spectral dispersion to obtain a spectrally dispersed interferogram for each scan (i.e. a two-dimensional array with interferometric fringes along the OPD axis which is perpendicular to the direction of spectral dispersion). The next step is the calculation of the Fourier transform at each spectral channel. In order to improve the signal-to-noise ratio, four spectral channels were averaged. Finally, the squared fringe amplitude for each spectral channel is obtained from the average power spectrum (i.e. average over all interferograms) at the corresponding fringe frequency. Division of the squared fringe amplitude by the photometric flux gives the squared raw visibility. For further details
Table 3. Summary of VINCI observations of RR Sco: date, modified Julian Date (MJD), time of observation (Universal Time = UTC), projected baseline length $B_{\mathrm{p}}$, and position angle of the projected baseline on the sky (PA).

\begin{tabular}{ccccc}
\hline \hline Date & MJD & $t_{\text {obs }}[\mathrm{UTC}]$ & $B_{\mathrm{p}}[\mathrm{m}]$ & ${\mathrm{PA}\left[{ }^{\circ}\right]}$ \\
\hline 2003 Jul. 10 & 52831.098 & $02: 20: 52$ & 16.0 & 70.5 \\
2003 Jul. 10 & 52831.103 & $02: 28: 10$ & 16.0 & 71.4 \\
2003 Jul. 10 & 52831.108 & $02: 35: 10$ & 16.0 & 72.3 \\
2003 Jul. 11 & 52832.032 & $00: 45: 32$ & 15.3 & 57.5 \\
2003 Jul. 11 & 52832.045 & $01: 05: 27$ & 15.6 & 60.7 \\
\hline
\end{tabular}

on the data reduction, we refer to the description in Leinert et al. (2004).

In addition to the MIDI measurements, we employed a total of five $K$-band VLTI VINCI observations of RR Sco which are publicly available in the ESO archive and were carried out on 2003 July 10 and 2003 July 11, roughly three weeks after the MIDI SDT observations. The two VLTI siderostats on stations E0 and G0 were used, forming a baseline length of $16 \mathrm{~m}$. A summary of these VINCI measurements are given in Table 3. Additional VINCI data of RR Sco obtained on 2003 June 30 and 2003 July 8 were found to be of poor quality and were not used. For the calibration of the VINCI data, we used the calibrator stars 58 Hya, 70 Aql, $\gamma$ Scl, HR 4546, HR 6862, HR 8090, $\tau$ Sgr, and $\epsilon$ Oph with limb-darkened diameters given in Bordé et al. (2002). The effective wavelength of the calibrator stars is $2.185 \mu \mathrm{m}$ and that of RR Sco is $2.192 \mu \mathrm{m}$, for adopted effective temperatures of $\sim 4000 \mathrm{~K}$ and $2500 \mathrm{~K}$, respectively. Mean coherence factors were obtained for each series of interferograms obtained for RR Sco with the VINCI data reduction software version 3.0 (Kervella et al. 2004), and we used the results based on the wavelet transform to derive the power spectral density instead of the classical Fourier analysis. Calibrated squared visibility values of RR Sco were obtained by calibrating the coherence factors as described in Wittkowski et al. (2004), with a time kernel of $6 \mathrm{~h}$ to convolve the measured transfer function.

\section{Results}

\subsection{MIDI measurements}

Figure 1a shows the calibrated visibilities of RR Sco for the seven data sets over the wavelength range from 8 to $13 \mu \mathrm{m}$. The visibilities of all the seven data sets of RR Sco show a distinct wavelength dependency: a gradual increase from 8 to $10 \mu \mathrm{m}$ and a roughly constant part longward of $10 \mu \mathrm{m}$. The difference in the visibility values at the same wavelength is mainly due to the different projected baseline lengths of the observations. To derive the calibrated object visibilities shown in Fig. 1a, we calibrated each raw data set of RR Sco with the instrumental transfer function values derived from each calibrator data set obtained on the same night (see Table 2) and calculated the average of the individual visibility values.

The errors of the calibrated visibilities consist of the following major contributions. Firstly, since chopping was not 

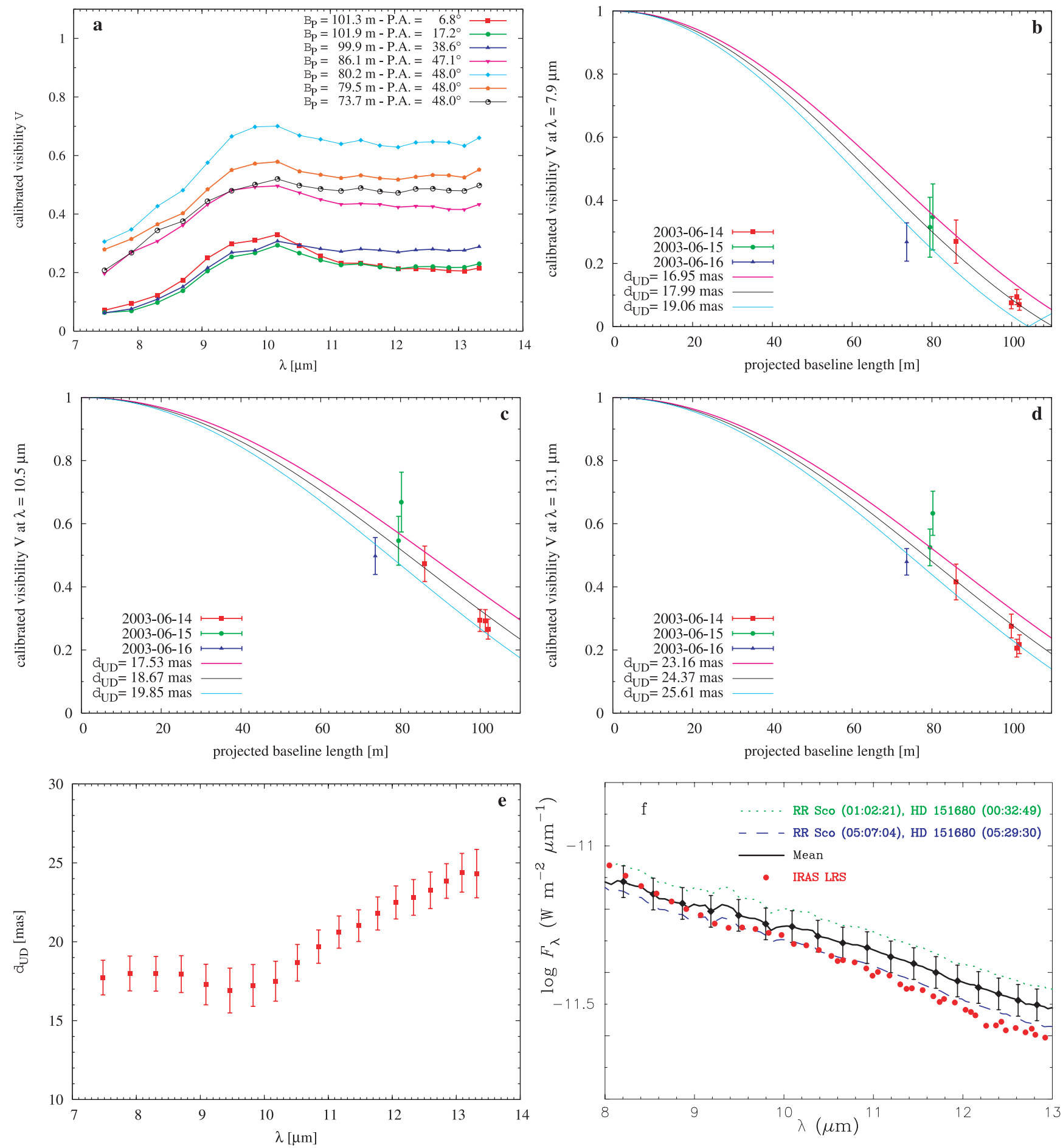

Fig. 1. a) Visibility as a function of wavelength for different projected baseline lengths ranging from $74 \mathrm{~m}$ to $102 \mathrm{~m}$. For all seven data sets of RR Sco the plot shows the visibility averaged over all calibrators of the corresponding night (see Table 2). All curves show a similar shape: a gradually increasing part shortward of $10 \mu \mathrm{m}$ and a roughly constant part longward of $10 \mu \mathrm{m}$. For the sake of clarity, error bars are omitted in this panel. b) Visibility as a function of the projected baseline for $\lambda=7.9 \mu \mathrm{m}$. In this panel, the error bars of the visibilities are shown. In addition, three uniform-disk fits are shown, representing the best-fitting model as well as a lower and an upper boundary fit curve which are used for estimating the error of the diameter. As the figure shows, the data can be fitted with a uniform disk with a diameter of $d_{\text {UD }}=18.0 \pm$ 1.07 mas. c) Same as b), but for $\lambda=10.5 \mu \mathrm{m}$. The uniform-disk fit yields $d_{\mathrm{UD}}=18.7 \pm 1.18$ mas. d) Same as b), but for $\lambda=13.1 \mu \mathrm{m}$. The uniform-disk fit yields $d_{\mathrm{UD}}=24.4 \pm 1.24$ mas. e) Uniform-disk diameter as a function of wavelength. The diameters are derived from uniform-disk fits using all seven averaged visibility data points of RR Sco, as exemplarily shown in b)-d) for three representative wavelengths. f) Absolutely calibrated spectra of RR Sco. The dashed and dotted lines represent the calibrated spectra of RR Sco derived from two data sets obtained on 2003 Jun. 14, using HD 151680 as a spectrophotometric standard star. The black solid line and the filled diamonds represent the mean of the above two spectra. The IRAS LRS is plotted with the filled circles. 
possible during the interferometric measurements, time-dependent atmospheric transmission (i.e. a possible change of atmospheric transmission between interferometric and the corresponding photometric observations) affects the visibilities. Secondly, an imperfect overlap of the two spectrally dispersed images on the detector introduces visibility errors. Thirdly, there is the error of the transfer function, which reflects the error of the raw visibility measurements of the calibrators and the uncertainties of their diameters. This error contribution was estimated from the standard deviation of all individual transfer function values obtained on each observing night. The estimated total errors of the calibrated object visibilities are shown in Figs. 1b-d.

Thanks to the projection effect, the projected baseline lengths of the seven data sets range from $\sim 70$ to $\sim 100 \mathrm{~m}$. Therefore, we plot the calibrated visibility from each data set against the projected baseline lengths. Figures $1 \mathrm{~b}-\mathrm{d}$ show these plots for three representative wavelengths of 7.9, 10.5, and $13.1 \mu \mathrm{m}$ together with uniform-disk fits for each wavelength, and in Fig. 1e we plot the results of these uniformdisk fits as a function of wavelength. As Fig. 1e shows, the wavelength dependence of the visibility translates into an approximately constant uniform-disk diameter of $\sim 18 \pm 1$ mas between 8 and $10 \mu \mathrm{m}$, with a very slight dip near $9.5 \mu \mathrm{m}$, and a steady increase of the diameter from 18 mas at $10 \mu \mathrm{m}$ to $\sim 24$ mas at $13 \mu \mathrm{m}$.

It should be noted that the first three data sets of the night 2003 Jun. 14 (\#1, \#2, and \#3 in Table 1) were obtained at approximately the same projected baseline length $\left(B_{\mathrm{p}} \sim 100 \mathrm{~m}\right)$ but with different position angles ranging from $7^{\circ}$ to $39^{\circ}$. However, given the uncertainties of the observed visibilities, we cannot draw a conclusion about the presence or absence of asymmetries, and therefore, as described above, we derived uniform-disk diameters from a fit of all available visibilities at each wavelength regardless of the position angles.

We also obtained the calibrated $N$-band spectrum of RR Sco from the MIDI data. Among the calibrators listed in Table 2, the absolutely calibrated spectra are available for HD 151680, HD 10380, HD 89388, and HD 139063 in Cohen et al. (1999). HD 151680 (K2.5III), in particular, is located close to RR Sco, and it was observed right before or after the observations of RR Sco at similar air masses on 2003 Jun. 14. Therefore, this calibrator can also serve as a spectrophotometric standard star. The other calibrators whose absolutely calibrated spectra are available were observed at quite different air masses from the observations of RR Sco, or at similar air masses, but at quite a low elevation $\left(\sim 30^{\circ}\right)$. The calibrated spectra of RR Sco were obtained by dividing the observed spectra of RR Sco by those of HD 151680 and then multiplying the absolutely calibrated spectrum of HD 151680 given by Cohen et al. (1999). Figure 1f shows the calibrated spectra of RR Sco. These spectra (dashed and dotted lines) are obtained for two data sets for RR Sco (\#1 and \#3 in Table 1) using two data sets for HD 151680 obtained close in time to \#1 and \#3. The mean of these two spectra is shown with the black solid line and the filled diamonds. The figure shows that the shapes of the two calibrated spectra of RR Sco are in agreement, while the difference in the absolute flux level is approximately $0.1 \mathrm{dex}$

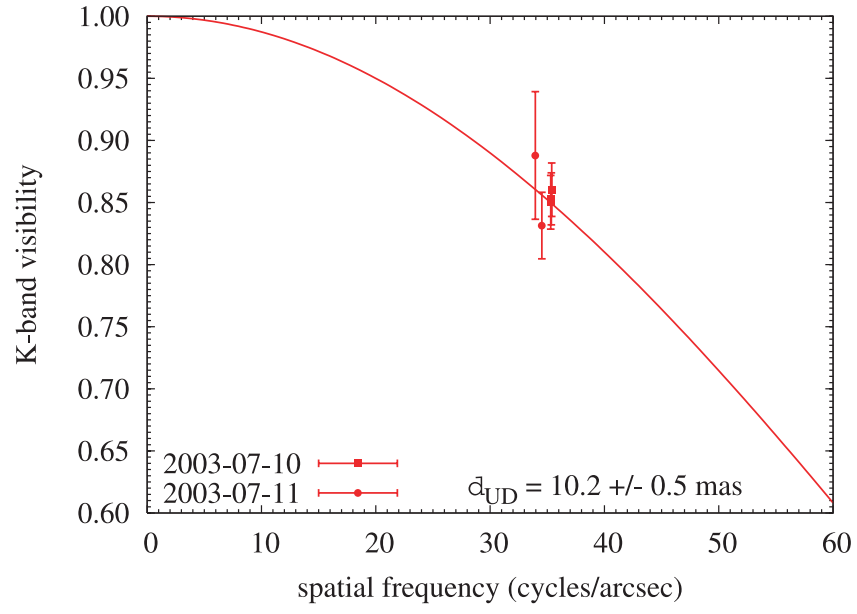

Fig. 2. $K$-band visibility of RR Sco as a function of spatial frequency. The figure shows the visibilities measured with VINCI on 2003 Jul. 10 and 2003 Jul. 11 together with a uniform-disk fit with $d_{\mathrm{UD}}=10.2$ mas.

(26\%), which we take as the uncertainty of the resulting calibrated spectrum of RR Sco. Figure 1f also shows a comparison with the IRAS LRS. Temporal variations of mid-infrared spectra of Mira variables are reported in Monnier et al. (1998), and a temporal variation as large as that seen between the MIDI spectrum and IRAS LRS of RR Sco is not unusual among Mira variables. However, given the accuracy of the MIDI calibrated spectrum, it is not clear whether the difference between the MIDI spectrum and the IRAS LRS can be attributed to a temporal variation of the spectrum. It should also be noted that IRAS LRS exhibits a slight bump at around $9.6 \mu \mathrm{m}$, which suggests dust emission. However, in the MIDI spectrum, the region around $9.6 \mu \mathrm{m}$ is least reliable due to the strong ozone absorption originating in the Earth's atmosphere, which makes it impossible to confirm the presence of this feature.

\section{2. $\mathrm{VINCI}$ measurements}

The result of our analysis of the VINCI observations of RR Sco is displayed in Fig. 2, where the $K$-band visibility is shown as a function of spatial frequency together with a uniform-disk fit. This fit was calculated as described in Wittkowski et al. (2004), using the VINCI broadband $K$ filter and by approximating the stellar spectrum with a blackbody spectrum with $T_{\mathrm{BB}}=2500 \mathrm{~K}$. The visibility errors shown in Fig. 2 include the standard deviation of the scan-to-scan scatter, the uncertainty of the adopted calibrator diameters, as well as the variation of the transfer function during the night. The fit reveals a uniform-disk diameter $d_{\mathrm{UD}}=10.2 \pm 0.5$ mas which is significantly smaller than the diameters derived with MIDI in the mid-infrared (see Fig. 1e). We checked the data for possible deviations from spherical symmetry, but such deviations could not be detected within the accuracy of the visibility measurements, as in the case of the MIDI observations. 


\section{Modeling of the observed visibility and the spectra}

The increase of the apparent diameter from the near-infrared toward longer wavelengths seems to be a common phenomenon in M (super)giants. Mennesson et al. (2002) and Chagnon et al. (2002) show that the $L^{\prime}$-band diameters of the oxygen-rich Miras and semiregular M giants are between $\sim 20 \%$ and $\sim 100 \%$ larger than those measured in the $K^{\prime}$ band. As a possible explanation for this increase of the apparent diameters, Mennesson et al. (2002) suggest the presence of optically thin gaseous layers extending to $\sim 3 R_{\star}$, and they also predict that the angular diameters increases further from the $L^{\prime}$ band to the $10 \mu \mathrm{m}$ region. A narrow-band interferometric observation in the $K$ band (Perrin et al. 2004a) demonstrates that the angular sizes of six Mira variables are larger in the $\mathrm{CO}$ and $\mathrm{H}_{2} \mathrm{O}$ bands compared to those in the continuum. Weiner et al. (2000, 2003a,b) measured the $11 \mu \mathrm{m}$ diameters of the Mira variables $o$ Cet, R Leo, and $\chi \mathrm{Cyg}$, as well as the M supergiants $\alpha$ Ori and $\alpha$ Her, using ISI. They found that the diameters of these Mira variables at $11 \mu \mathrm{m}$ are larger by a factor of $\sim 2$ than those measured in the $K$ band, while the diameters of the M supergiants at $11 \mu \mathrm{m}$ are larger by a factor of $\sim 1.3$ than those in the $K$ band. Perrin et al. (2004b) have shown that the $11.15 \mu \mathrm{m}$ diameters of $\alpha$ Ori and $\alpha$ Her measured by Weiner et al. (2000) can be reproduced by an extended gaseous envelope model with its optical depth as a free parameter. Ohnaka $(2004 a, b)$ further shows that the observed increase of the angular diameter from the nearinfrared to the $11 \mu \mathrm{m}$ region and the high-resolution $11 \mu \mathrm{m}$ spectra of the Mira variables as well as the $M$ supergiants observed by Weiner et al. (2000, 2003a,b) can be simultaneously reproduced by a warm water vapor envelope extending to 1.4-2.5 $R_{\star}$, with temperatures of $1200-2000 \mathrm{~K}$ and $\mathrm{H}_{2} \mathrm{O}$ column densities of the order of $10^{20}-10^{21} \mathrm{~cm}^{-2}$. The presence of such a warm molecular envelope has been revealed by recent analyses of infrared molecular spectra in Mira variables as well as semiregular $\mathrm{M}$ giants. The analyses of the spectra obtained with ISO/SWS, in particular, have revealed that the spectra of Mira variables are well reproduced by the water vapor envelope extending to $2-4 R_{\star}$ with gas temperatures of $1000-2000 \mathrm{~K}$ and column densities of $10^{20}-10^{22} \mathrm{~cm}^{-2}$ (e.g., Tsuji et al. 1997; Yamamura et al. 1999; Matsuura et al. 2002). In the wavelength region observed with MIDI, the strong purerotation lines of $\mathrm{H}_{2} \mathrm{O}$ are expected to be ubiquitous in the region between 8 and $13 \mu \mathrm{m}$, together with the fundamental bands of $\mathrm{SiO}$ located between 8 and $10 \mu \mathrm{m}$. Therefore, it is well possible that the increase of the uniform-disk diameter of RR Sco from the $K$ band to the $N$ band can be explained by the presence of a warm molecular envelope consisting of $\mathrm{H}_{2} \mathrm{O}$ and $\mathrm{SiO}$.

\subsection{Warm $\mathrm{H}_{2} \mathrm{O}+\mathrm{SiO}$ envelope}

In the present work, we attempt to interpret the observed visibility using a simple model of the warm molecular envelope presented by Ohnaka (2004a). We approximate the star with a blackbody of $3000 \mathrm{~K}$ and a radius $R_{\star}$. The star is surrounded by a warm molecular envelope consisting of $\mathrm{H}_{2} \mathrm{O}$ and $\mathrm{SiO}$ gas with a constant temperature and density, extending to $R_{\mathrm{mol}}$.
The inner radius of the molecular envelope is set to be equal to $R_{\star}$. The input parameters of our model are the outer radius and the temperature of the molecular envelope $\left(R_{\mathrm{mol}}\right.$ and $T_{\text {mol }}$, respectively) as well as the column densities of $\mathrm{H}_{2} \mathrm{O}$ and $\mathrm{SiO}\left(N_{\mathrm{H}_{2} \mathrm{O}}\right.$ and $N_{\mathrm{SiO}}$, respectively) in the radial direction. While two-layer models with hot and cool $\mathrm{H}_{2} \mathrm{O}$ layers are often used in the analyses of the ISO spectra of Mira variables (e.g., Yamamura et al. 1999; Matsuura et al. 2002), we prefer to adopt a one-layer model in the present work, because spectroscopic information enough to constrain so many free parameters (the gas temperature, $\mathrm{H}_{2} \mathrm{O}$ and $\mathrm{SiO}$ column densities, and the radius of each of the hot and cool layers) is not available for RR Sco.

We estimate the stellar radius $R_{\star}$ from the $K$-band uniformdisk diameter measured with VINCI. It has been pointed out that the uniform disk is not a good approximation for extended atmospheres of Miras, and that the $\mathrm{H}_{2} \mathrm{O}$ and $\mathrm{CO}$ absorption in the $K$ band also makes the apparent diameter appear larger than the continuum diameter (Perrin et al. 1999). Woodruff et al. (2004) analyzed the VINCI measurements of the prototypical Mira $o$ Cet at phases from 0.13 to 0.40 . They derived the Rosseland radius as well as the continuum radius at $1.04 \mu \mathrm{m}$ at each phase by fitting the observed visibilities with theoretical ones predicted by dynamical model atmospheres. Their analysis shows that the uniform-disk diameter of $o$ Cet at phase 0.4 is 33.3 mas, while the continuum diameter at $1.04 \mu \mathrm{m}$ at the same phase is 29.5 mas, which is $13 \%$ smaller than the uniform-disk diameter. We apply this conversion factor obtained for $o$ Cet at phase 0.4 , which is the closest to the phase 0.6 at the time of the MIDI observation of RR Sco, to the $K$-band uniform-disk diameter of 10.2 mas obtained with VINCI. This results in a $1.04 \mu \mathrm{m}$ continuum diameter of 9.0 mas, which corresponds to a radius of 4.5 mas. We adopt this $1.04 \mu \mathrm{m}$ continuum radius as the angular radius corresponding to the stellar radius $R_{\star}$.

We first calculate the line opacity due to $\mathrm{H}_{2} \mathrm{O}$ and $\mathrm{SiO}$ in the wavelength range between 8 and $13 \mu \mathrm{m}$. We adopt a Gaussian line profile with a $F W H M$ of $5 \mathrm{~km} \mathrm{~s}^{-1}$, which represents the thermal and (micro-)turbulent velocities in the atmosphere of RR Sco, and assume that the molecular gas is in local thermodynamical equilibrium. The line list of $\mathrm{H}_{2} \mathrm{O}$ was taken from the HITEMP database (Rothman 1997), while the line list of the fundamental bands of ${ }^{28} \mathrm{SiO}$ was generated from the Dunham coefficients given by Lovas et al. (1981) and the dipole moment matrix elements derived by Tipping \& Chackerian (1981). The line positions of ${ }^{29} \mathrm{SiO}$ and ${ }^{30} \mathrm{SiO}$ are computed by applying the usual mass conversion factors, as described in Tsuji et al. (1994). It should be noted, however, that the transition probabilities of the $\mathrm{SiO}$ fundamental and first-overtone bands are still controversial. For example, Langhoff \& Bauschlicher (1993) obtained an Einstein coefficient $A_{10}=7.25 \mathrm{~s}^{-1}$, while Tipping \& Chackerian (1981) obtained $A_{10}=5.166 \mathrm{~s}^{-1}$. Recently, Drira et al. (1997) have derived a value of $A_{10}=6.61 \mathrm{~s}^{-1}$, which lies between the values calculated by Tipping \& Chackerian (1981) and Langhoff \& Bauschlicher (1993). We include this uncertainty of the transition probabilities of the $\mathrm{SiO}$ fundamental bands in the resulting uncertainty of the column density of SiO. No quantitative analysis of silicon isotope ratios in Mira variables has been carried out up to now, but Tsuji et al. (1994) analyzed the silicon isotope ratios in non-Mira $\mathrm{M}$ giants as 
well as in $\mathrm{M}$ supergiants. They derived ${ }^{28} \mathrm{Si} /{ }^{29} \mathrm{Si}$ ratios ranging from 13 to 20 and ${ }^{28} \mathrm{Si} /{ }^{30} \mathrm{Si}$ ratios ranging from 17 to 28 for $\mathrm{M}$ giants. For our models, we use isotope ratios of 15 and 20 for ${ }^{28} \mathrm{Si} /{ }^{29} \mathrm{Si}$ and ${ }^{28} \mathrm{Si} /{ }^{30} \mathrm{Si}$, respectively. Once the line opacity has been calculated, the monochromatic intensity profile, monochromatic visibility squared, and emergent flux are calculated with a wavenumber interval of $0.01 \mathrm{~cm}^{-1}$. The calculated monochromatic visibility squared is then spectrally convolved to match the spectral resolution of 30 , which was used in the SDT observations. Finally, the uniform-disk diameter is derived in the same manner as the observed uniform diskdiameter, that is, the uniform-disk diameter is derived from the spectrally convolved visibility for the projected baseline lengths used in the SDT observations.

We calculate models for $T_{\text {mol }}$ between 1000 and $2000 \mathrm{~K}$, $R_{\text {mol }}$ between 1.5 and $3 R_{\star}$, and $N_{\mathrm{H}_{2} \mathrm{O}}$ and $N_{\mathrm{SiO}}$ between $10^{19}$ and $10^{22} \mathrm{~cm}^{-2}$, with intervals of $\Delta T_{\mathrm{mol}}=100 \mathrm{~K}, \Delta R_{\mathrm{mol}}=$ $0.1 R_{\star}$, and $\Delta \log N_{\mathrm{H}_{2} \mathrm{O}}=\Delta \log N_{\mathrm{SiO}}=0.5$, respectively, searching for a parameter set which can best reproduce the observed $N$-band visibility and spectrum of RR Sco. As we will discuss in Sect. 4.3, comparison with the observed $K$-band visibility is also taken into account as a constraint on the parameters. Figure 3 shows the visibility, the uniform-disk diameter, and the spectrum predicted from a model with $T_{\mathrm{mol}}=$ $1400 \mathrm{~K}, R_{\mathrm{mol}}=2.3 R_{\star}, N_{\mathrm{H}_{2} \mathrm{O}}=3 \times 10^{21} \mathrm{~cm}^{-2}$, and $N_{\mathrm{SiO}}=$ $1 \times 10^{20} \mathrm{~cm}^{-2}$, which is one of the models that can well reproduce the observed visibility and the uniform-disk diameter shortward of $\sim 9.5 \mu \mathrm{m}$. While the uncertainty of the $\mathrm{H}_{2} \mathrm{O}$ column density is roughly a factor of 2 , the $\mathrm{SiO}$ column density cannot be well constrained from the present data, because the effects of the $\mathrm{SiO}$ fundamental bands on the angular diameter as well as on the spectrum tend to be masked by stronger $\mathrm{H}_{2} \mathrm{O}$ lines, and therefore, the uncertainty of the derived $\mathrm{SiO}$ column density is as large as a factor of 10 . The predicted visibilities shown in Fig. 3 are calculated for baseline lengths of $100 \mathrm{~m}$ and $74 \mathrm{~m}$. The figure reveals that the visibility as well as the uniform-disk diameter between 8 and $\sim 9.5 \mu \mathrm{m}$ is well reproduced. The molecular envelope is optically thick between 8 and $13 \mu \mathrm{m}\left(\tau_{\mathrm{H}_{2} \mathrm{O}+\mathrm{SiO}} \simeq 1-10\right)$, which causes the apparent diameter to be significantly larger than the continuum diameter.

However, Fig. 3 demonstrates that the agreement with the observed visibility as well as the uniform-disk diameter becomes unsatisfactory longward of $\sim 9.5 \mu \mathrm{m}$. The discrepancy is also obvious in Fig. 3c, which shows a comparison between the observed spectrum of RR Sco and the calculated spectrum, which is spectrally convolved with a resolution of 30 to match the observation. The predicted spectrum is much steeper than the observed one, which shows a very small bump starting at around $9.5 \mu \mathrm{m}$ (see IRAS LRS plotted in Fig. 1f), indicating the presence of dust grains. Furthermore, the location of the small bump coincides with the wavelength where model and observation start to show a significant discrepancy in the comparison of the visibility and uniform-disk diameter. Therefore, the presence of an optically thin dust shell in RR Sco can be responsible for the increase of the apparent diameter longward of $9.5 \mu \mathrm{m}$ as well as the discrepancy between the observed and calculated spectra.
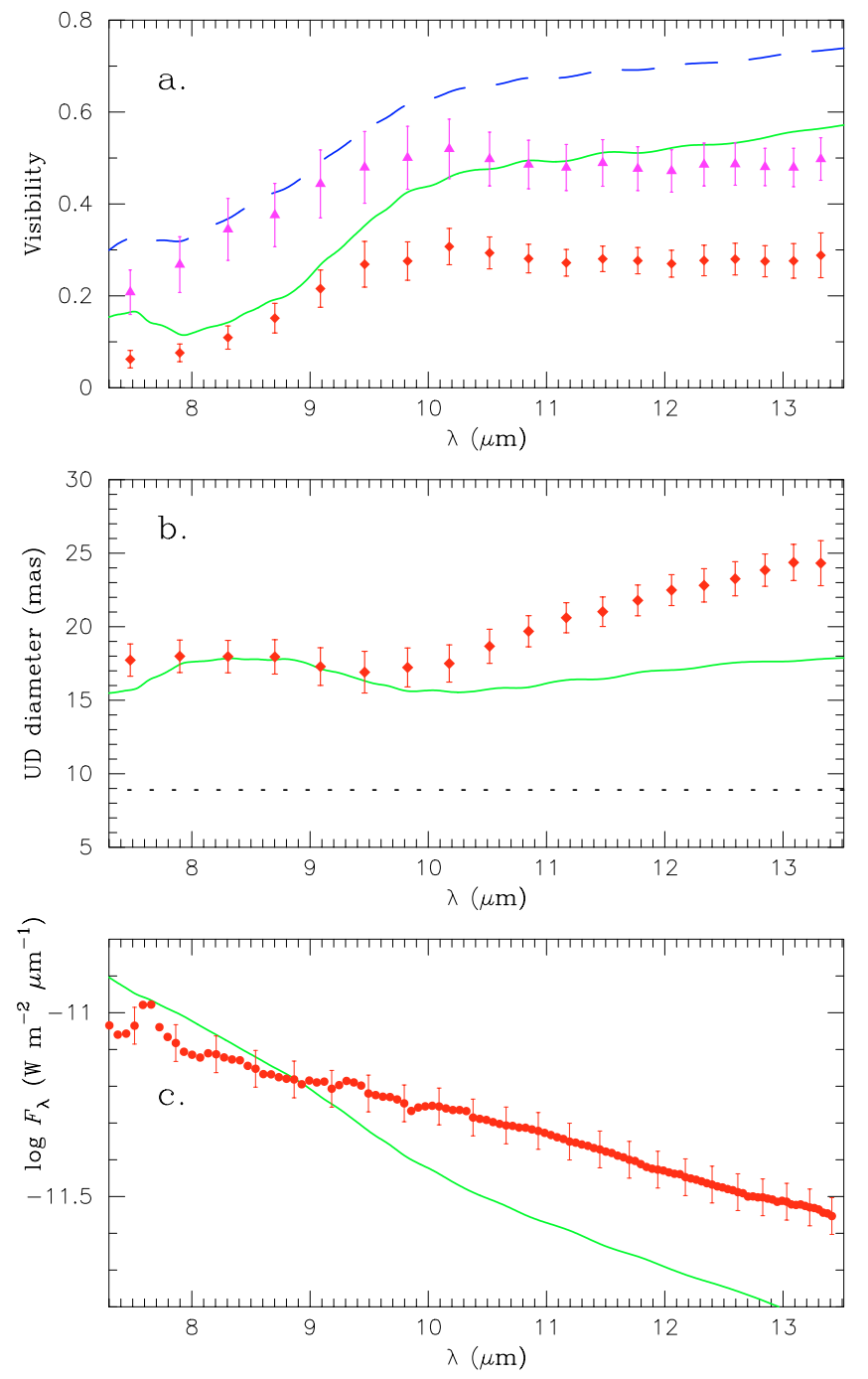

Fig. 3. Comparison between the observed visibility a), uniform-disk diameter b), and spectrum c) and those predicted by a model for RR Sco with the warm molecular $\left(\mathrm{H}_{2} \mathrm{O}+\mathrm{SiO}\right)$ envelope alone. The parameters of the molecular envelope are $T_{\mathrm{mol}}=1400 \mathrm{~K}, R_{\mathrm{mol}}=2.3 R_{\star}$, $N_{\mathrm{H}_{2} \mathrm{O}}=3 \times 10^{21} \mathrm{~cm}^{-2}$, and $N_{\mathrm{SiO}}=1 \times 10^{20} \mathrm{~cm}^{-2}$. a) The filled diamonds and triangles represent the visibilities observed with projected baseline lengths of $99.9 \mathrm{~m}$ (data set \#3) and $73.7 \mathrm{~m}$ (data set \#7), respectively, while the corresponding predicted visibilities are represented by the solid and dashed lines, respectively. b) The filled diamonds represent the observed values, which are the same as in Fig. 1e, while the solid line represents the uniform-disk diameter predicted for a projected baseline length of $100 \mathrm{~m}$. The dotted line represents the continuum angular diameter assumed in the calculation. c) The filled circles represent the calibrated spectrum of RR Sco obtained from the observations on 2003 Jun. 14 (the same as the black solid line in Fig. 1f). The solid line represents the spectrum predicted from the model.

\subsection{Optically thin dust shell}

The dust emission in RR Sco is very weak, and the overall shape of the MIDI spectrum as well as IRAS LRS resembles those of the intermediate class objects modeled by Lorenz-Martins \& Pompeia (2000), although the dust emission feature at $\sim 9.6 \mu \mathrm{m}$ in RR Sco is even weaker than that in the 
intermediate objects they studied. These intermediate objects lie between objects with clear silicate features peaked at 9.8 and $18 \mu \mathrm{m}$ (Sil class) and those with a broad emission feature from 9 to $15 \mu \mathrm{m}$ without a well defined peak (Broad class), which Lorenz-Martins \& Pompeia (2000) modeled well with corundum $\left(\mathrm{Al}_{2} \mathrm{O}_{3}\right)$ grains. They found that the spectral energy distributions (SEDs) of the intermediate objects can be reproduced by optically thin dust shell models made of two grain species, that is, corundum and silicate. They derived ratios of corundum to silicate ranging from 0.6 to 0.9 and grain sizes of $0.1-0.2 \mu \mathrm{m}$.

We calculate the contribution of the optically thin dust shell based on a simple, spherical model consisting of a mixture of silicate and corundum dust. We first calculate the opacity of silicate and corundum in the Mie theory, assuming a single grain size, $a=0.1 \mu \mathrm{m}$, and using the code published by Bohren $\&$ Huffman (1983). As in Lorenz-Martins \& Pompeia (2000), we calculate the opacity of silicate grains from the dielectric function obtained by David \& Pégourié (1995). As for the opacity of corundum dust, Lorenz-Martins \& Pompeia (2000) show that the use of the alumina grains obtained by Koike et al. (1995) for $\lambda<8 \mu \mathrm{m}$ and the porous amorphous $\mathrm{Al}_{2} \mathrm{O}_{3}$ obtained by Begemann et al. (1997) for $\lambda>8 \mu \mathrm{m}$ can reproduce the IRAS LRS of their intermediate class objects quite well. We also adopt this approach for the opacity of corundum grains. Next, the averaged opacity of the mixture of silicate and corundum is calculated with different ratios of the two components. In the present work, we adopt ratios of silicate to the sum of the two components ranging from 0.1 to 0.7 . Assuming a dust density distribution proportional to $r^{-2}$, the temperature distribution in the dust shell is calculated from thermal balance in the optically thin limit, as described in Ohnaka et al. (2001). The input parameters are the temperature at the inner boundary and the optical depth of the dust shell. Then the location of the inner boundary can be known from the temperature distribution calculated above. The outer radius of the dust shell is set to be $100 R_{\star}$, but it does not have a major effect on the resulting flux and intensity profile in the relevant spectral range.

We add this dust shell to the warm $\mathrm{H}_{2} \mathrm{O}+\mathrm{SiO}$ envelope model discussed above, and the total flux and the intensity profile are the sum of the contributions of these two components. Figure 4 shows a comparison of the observed visibility, uniform-disk diameter, and spectrum with those predicted from the best-fit model. We find that the observed spectrum and visibilities can be best reproduced with a dust mixture of $20 \%$ silicate and $80 \%$ corundum. The optical depth of the dust shell is derived to be $0.025 \pm 0.01$ at $10 \mu \mathrm{m}$, which translates into 0.23 in the visual. The inner boundary of the dust shell is found to be $7.5 R_{\star}$ with a dust temperature of $700 \pm 200 \mathrm{~K}$. The radius of the inner boundary as well as the dust temperature there is in rough agreement with those derived for the intermediate class objects studied by Lorenz-Martins \& Pompeia (2000), while the ratio of corundum grains to silicate grains derived here $(\mathrm{Cor} / \mathrm{Sil}=4.0)$ is significantly larger than the values they derived $(\mathrm{Cor} / \mathrm{Sil}=0.6-0.9)$. This result suggests that RR Sco may not be a typical intermediate class object but an object between the intermediate class and the broad class, which is consistent with the very weak $9.6 \mu \mathrm{m}$ feature in RR Sco.
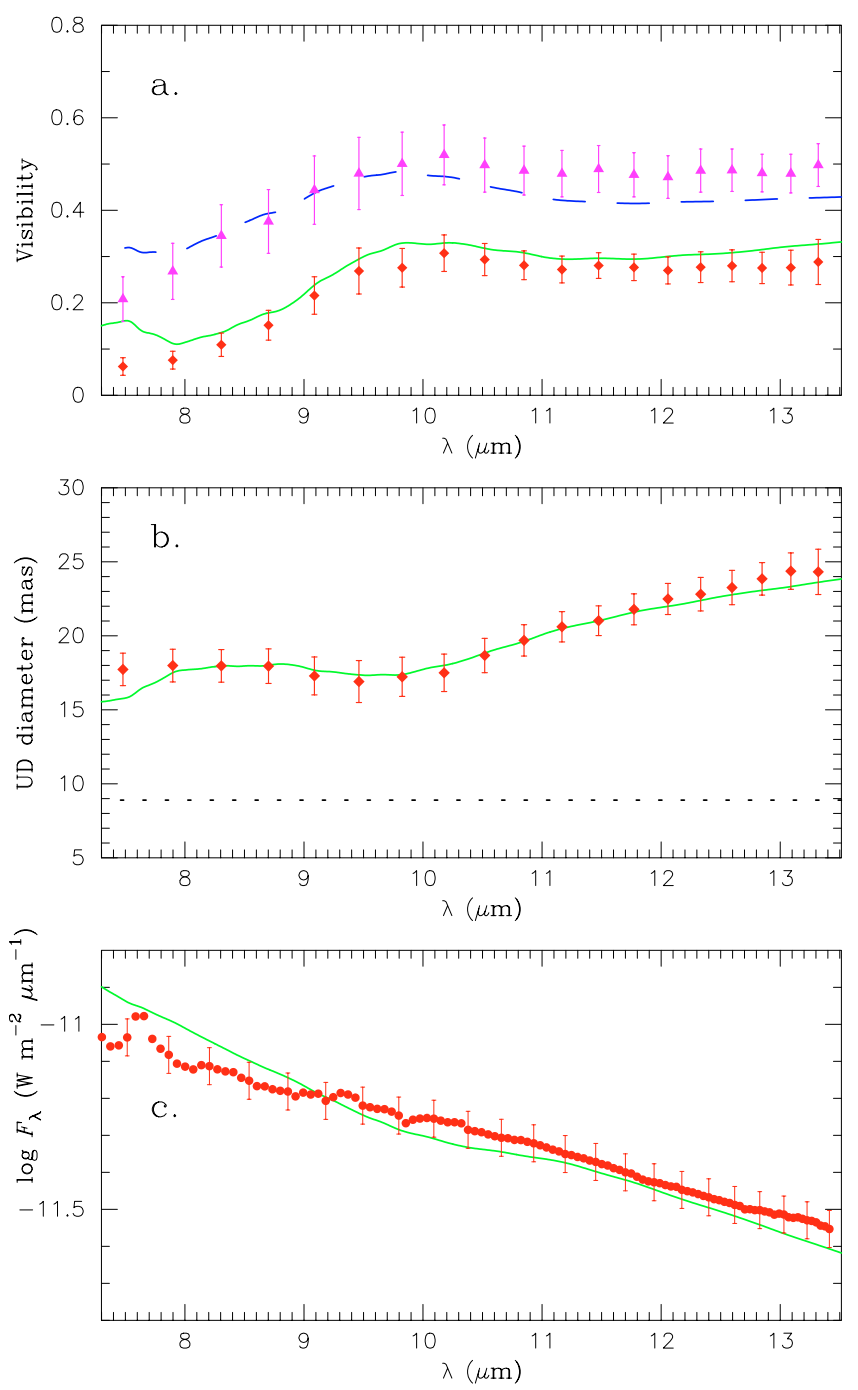

Fig. 4. Comparison between the observed visibility a), uniform-disk diameter b), and spectrum c) and those predicted by the best-fit model for RR Sco. The parameters of the $\mathrm{H}_{2} \mathrm{O}+\mathrm{SiO}$ envelope are $T_{\text {mol }}=$ $1400 \mathrm{~K}, R_{\mathrm{mol}}=2.3 R_{\star}, N_{\mathrm{H}_{2} \mathrm{O}}=3 \times 10^{21} \mathrm{~cm}^{-2}$, and $N_{\mathrm{SiO}}=1 \times$ $10^{20} \mathrm{~cm}^{-2}$. The dust shell has an inner radius of $7.5 R_{\star}$ with a temperature of $700 \mathrm{~K}$ and an optical depth of 0.025 at $10 \mu \mathrm{m}$. a) The diamonds and triangles represent the visibilities observed with projected baseline lengths of $99.9 \mathrm{~m}$ (data set \#3) and $73.7 \mathrm{~m}$ (data set \#7), respectively, while the corresponding predicted visibilities are represented with the solid and dashed lines, respectively. b) The filled diamonds represent the observed values, which are the same as in Fig. 1e, while the solid line represents the uniform-disk diameter predicted for a projected baseline length of $100 \mathrm{~m}$. The dotted line represents the continuum angular diameter assumed in the calculation. c) The filled circles represent the calibrated spectrum of RR Sco obtained from the observations on 2003 Jun. 14 (the same as the black solid line in Fig. 1f). The solid line represents the spectrum predicted from the best-fit model.

The parameters of the warm $\mathrm{H}_{2} \mathrm{O}+\mathrm{SiO}$ envelope are $T_{\text {mol }}=$ $1400 \pm 100 \mathrm{~K}, R_{\mathrm{mol}}=2.3 \pm 0.2 R_{\star}, N_{\mathrm{H}_{2} \mathrm{O}}=3 \times 10^{21} \mathrm{~cm}^{-2}$, and $N_{\mathrm{SiO}}=1 \times 10^{20} \mathrm{~cm}^{-2}$, respectively. The uncertainties of the $\mathrm{H}_{2} \mathrm{O}$ and $\mathrm{SiO}$ column densities are roughly a factor of 2 and 10 , respectively. Figure 4 shows that the observed visibility and uniform-disk diameter are well reproduced from 8 to $13 \mu \mathrm{m}$. 

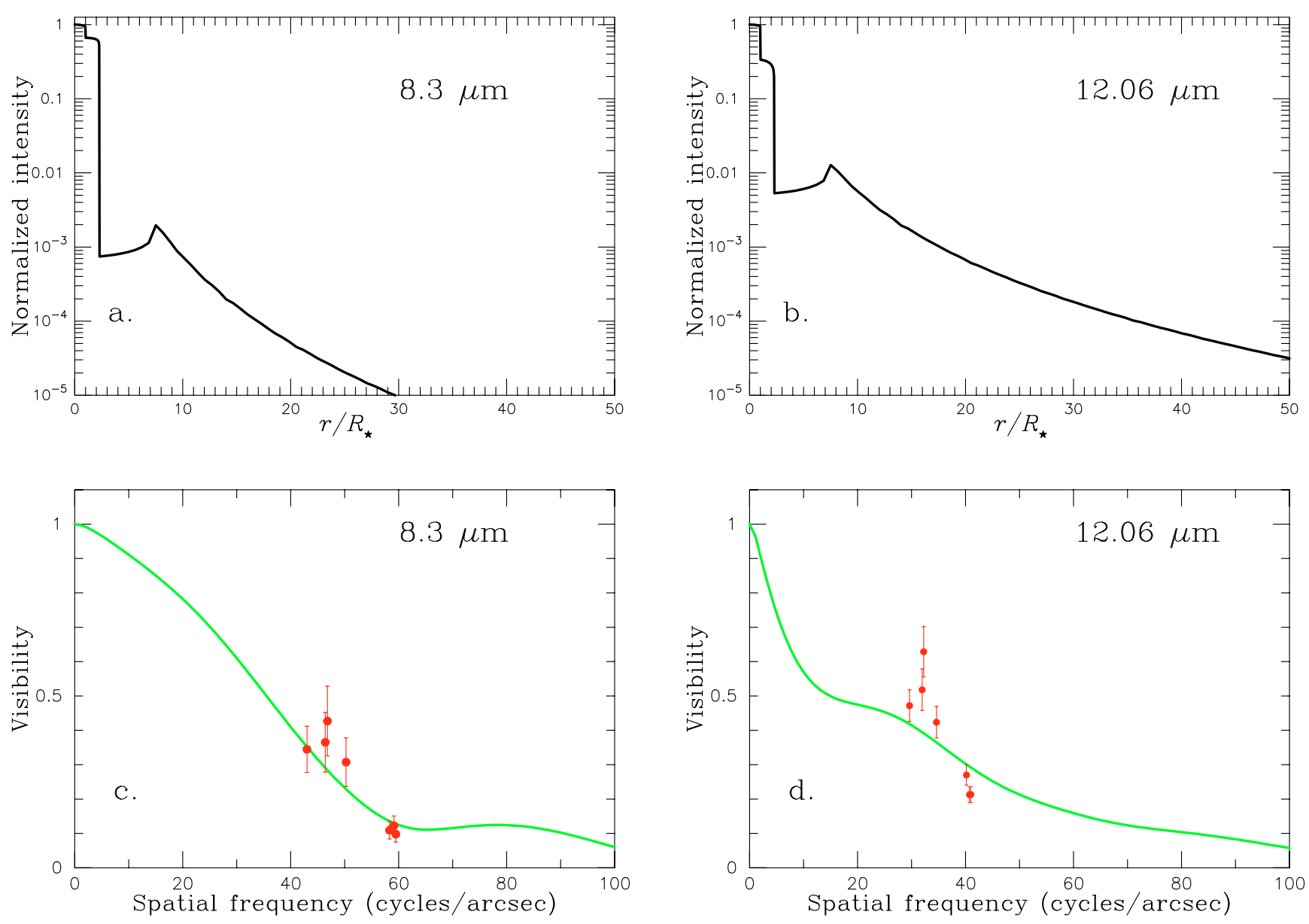

Fig. 5. Intensity profiles at 8.3 and $12.06 \mu \mathrm{m}$ predicted from the best-fit model for RR Sco (a) and b)), together with the corresponding visibilities (c) and d)). The filled circles in c) and d) represent the observed visibilities.

The predicted spectrum is now also in good agreement with the observed MIDI spectrum. The temperatures and column densities of $\mathrm{H}_{2} \mathrm{O}$ and $\mathrm{SiO}$ gas derived here are in agreement with those previously derived for other oxygen-rich Mira variables by Yamamura et al. (1999) and Matsuura et al. (2002). It is also worth noting that the radius of the molecular layer derived here, $2.3 R_{\star}$, is consistent with the result obtained by Cotton et al. (2004), who found that the $\mathrm{SiO}$ maser toward seven Mira variables originates in the region of $1.7-2.8 R_{\star}$.

Figure 5 shows intensity profiles at 8.3 and $12.1 \mu \mathrm{m}$ predicted from the above best-fit model, together with the spectrally convolved visibilities at these wavelengths to match the spectral resolution used in our MIDI observations. At $8.3 \mu \mathrm{m}$, the flux contribution of the dust shell is approximately $5 \%$. Therefore, the flux is dominated by emission from the stellar disk and the warm $\mathrm{H}_{2} \mathrm{O}+\mathrm{SiO}$ envelope. As Fig. 5 illustrates, the intensity profile is the sum of the warm $\mathrm{H}_{2} \mathrm{O}+\mathrm{SiO}$ envelope, which can be seen as a "step" at $2.3 R_{\star}$, and the optically thin dust shell, which shows limb-brightening at the inner boundary of $7.5 R_{\star}$. It is noteworthy that the intensity profile predicted by this simple model for the warm $\mathrm{H}_{2} \mathrm{O}+\mathrm{SiO}$ envelope resembles that predicted by dynamical models of Mira variables. Woodruff et al. (2004) demonstrate that the $K$-band visibility of $o$ Cet at phase 0.36 can be well fitted with an intensity profile which shows a significant "protrusion" caused by water vapor layers. It implies the possibility that the large-amplitude pulsation in Mira variables is responsible for the warm molecular envelope in these objects, while the formation mechanism of the warm molecular envelope in semiregular and irregular M (super)giants, in which the variability amplitudes are much smaller, remains unclear. The $8.3 \mu \mathrm{m}$ visibility calculated from this intensity profile shows fair agreement with those observed.

At $12 \mu \mathrm{m}$, the flux contribution of the dust shell is approximately $40 \%$. The visibility at this wavelength can roughly reproduce the observed visibility, but the agreement is poorer compared to the case for $8.3 \mu \mathrm{m}$. This can be due to the nonoptimal calibration of the observed data mentioned in Sect. 3.1, as well as the simplifications and/or assumptions adopted in our modeling of the dust shell, such as the averaged absorption coefficients for a mixture of two different species of dust and uncertainties in the dust opacity.

\subsection{Effects of the warm molecular envelope on the angular diameter in the near-infrared}

We have shown that optically thick emission due to $\mathrm{H}_{2} \mathrm{O}$ and $\mathrm{SiO}$ in the extended envelope can explain the increase of the angular diameter from the $K$ band to the $N$ band as well as the wavelength dependence of the angular diameter inside the $N$ band. However, if such a dense molecular envelope is 
present, it can also affect the angular diameter measured in the $K$ band. In fact, Perrin et al. (2004a) demonstrate that the angular diameters in six Mira variables are larger in the $\mathrm{CO}$ and $\mathrm{H}_{2} \mathrm{O}$ bands. If $K$-band diameters predicted by our model of the water vapor envelope are not in agreement with that measured with VINCI, our estimate of the stellar continuum diameter based on the VINCI measurements as well as the fit to the observed $N$-band visibility can no longer be justified. Therefore, we used the $K$-band uniform-disk diameter measured with VINCI as an additional constraint on deriving the parameters of the $\mathrm{H}_{2} \mathrm{O}+\mathrm{SiO}$ envelope above, and we describe here the calculation in the $K$ band.

Using the model described above, we calculated the $K$-band diameter with the absorption due to $\mathrm{H}_{2} \mathrm{O}$ and $\mathrm{CO}$ taken into account. For the $\mathrm{H}_{2} \mathrm{O}$ column density, we adopt the same values used for calculations of the $N$-band spectra and visibilities, while for $\mathrm{CO}$ we tentatively adopt a column density of $10^{22} \mathrm{~cm}^{-2}$. The CO first overtone bands are located longward of $2.3 \mu \mathrm{m}$, which is close to the longer edge of the $K$ band, and therefore, the effect of the uncertainty of the CO column density on the angular diameter measured with the broadband $K$ filter would be minor compared to that of $\mathrm{H}_{2} \mathrm{O}$. The stellar continuum diameter is assumed to be 9.0 mas, as in the above calculation for the $N$ band. The $K$-band uniform-disk diameter predicted by the best-fit model, which can well reproduce the observed $N$-band visibility and spectra, has turned out to be 10.6 mas for a projected baseline length of $16 \mathrm{~m}$, and this predicted value is in agreement with the $10.2 \pm 0.5$ mas measured with VINCI. This calculation lends support to our estimate of the stellar continuum diameter.

The wavelength dependence of the effect of the warm molecular envelope on the angular diameter can be explained as follows. The effects of emission from the warm molecular envelope on the angular diameter can be measured by the ratio between the emission from the star and the envelope, which is given by

$$
\frac{B_{\nu}\left(T_{\mathrm{mol}}\right) \cdot\left(1-\mathrm{e}^{-\tau_{v}}\right)}{B_{v}\left(T_{\mathrm{eff}}\right) \cdot \mathrm{e}^{-\tau_{v}}}
$$

The emission from the star and the warm molecular envelope is represented by the blackbodies of $T_{\mathrm{eff}}$ and $T_{\text {mol }}$, respectively, while $\tau_{v}$ represents the optical depth of the molecular envelope. For $T_{\text {eff }}=3000 \mathrm{~K}$ and $T_{\text {mol }}=1000-2000 \mathrm{~K}, B_{v}\left(T_{\text {mol }}\right) / B_{v}\left(T_{\text {eff }}\right)$ is small in the near-infrared and increases toward longer wavelengths. With optical depths of $0.1-1$ and $1-10$ in the $K$ and $N$ bands, respectively, $\left(1-\mathrm{e}^{-\tau_{\nu}}\right) / \mathrm{e}^{-\tau_{\nu}}$ is larger in the $N$ band than in the $K$ band. These two factors lead to an increase of the intensity contribution of the warm molecular envelope at longer wavelengths, which manifests itself as the increase of the angular diameter from the near-infrared to the mid-infrared.

\section{Concluding remarks}

We analyzed the VLTI MIDI observations of the oxygen-rich Mira star RR Sco carried out in June 2003 within the framework of the Science Demonstration Time program. We found that the visibility shows an increase between 8 and $10 \mu \mathrm{m}$, and the uniform-disk diameter in this wavelength range is roughly constant and approximately 18 mas, which is significantly larger than the $K$-band uniform-disk diameter of $10.2 \pm$ 0.5 mas measured with VLTI VINCI three weeks after the MIDI observations. Between 10 and $13 \mu \mathrm{m}$ the visibility remains nearly constant, and the uniform-disk diameter gradually increases from 18 to 24 mas.

We have demonstrated that this increase of the angular diameter from the $K$ band to the $N$ band as well as the observed $N$-band spectrum of RR Sco can be reproduced by our simple model of the warm molecular envelope consisting of $\mathrm{H}_{2} \mathrm{O}$ and $\mathrm{SiO}$. We have calculated the intensity profile and visibility using $\mathrm{H}_{2} \mathrm{O}$ and $\mathrm{SiO}$ line lists and shown that the optically thick emission due to $\mathrm{H}_{2} \mathrm{O}$ and $\mathrm{SiO}$ from the extended envelope causes the star to appear significantly larger than in the $K$ band. The temperature, the $\mathrm{H}_{2} \mathrm{O}$ and $\mathrm{SiO}$ column densities, and the radius of this warm molecular envelope are derived to be $1400 \mathrm{~K}, 3 \times 10^{21} \mathrm{~cm}^{-2}, 1 \times 10^{20} \mathrm{~cm}^{-2}$, and $2.3 R_{\star}$, respectively. The increase of the uniform-disk diameter longward of $10 \mu \mathrm{m}$ can be explained by the presence of an optically thin dust shell. Our model consisting of the warm $\mathrm{H}_{2} \mathrm{O}+\mathrm{SiO}$ envelope and an optically thin dust shell of silicate and corundum can reproduce the observed wavelength dependence of the uniform-disk diameter as well as the observed spectrum. A mixture of $20 \%$ silicate and $80 \%$ corundum is found to reproduce the observed spectrum reasonably well. The optical depth of the dust shell is derived to be 0.025 at $10 \mu \mathrm{m}$, and the inner boundary is at $7-8 R_{\star}$ with a temperature of $\sim 700 \mathrm{~K}$.

The present analysis demonstrates that spectrointerferometry in the mid-infrared is useful for studying the warm molecular envelope, whose properties have mostly been derived from spectroscopic observations up to now. We have shown that the measurement of the wavelength dependence of the angular size over molecular as well as dust spectral features in the mid-infrared provides direct information on the geometrical extension of the warm molecular envelope and such information, combined with spectral data, can help put more constraints on physical properties of the warm molecular envelope. Molecular features in the near-infrared such as the $\mathrm{H}_{2} \mathrm{O}$ vib-rotation lines and the $\mathrm{CO}$ first overtone bands will provide us with further important information about physics and chemistry in this warm molecular envelope. High-resolution spectro-interferometry with VLTI AMBER, which will be operated in the $J, H$, and $K$ bands with a spectral resolution of up to 10000 , is well suited for studying these molecular features and, together with MIDI, is expected to help us obtain a more comprehensive picture of the outer atmosphere of AGB stars.

Acknowledgements. The authors would like to thank the referee, Dr. B. Mennesson, for his valuable and constructive comments.

\section{References}

Begemann, B., Dorschner, J., Henning, Th., \& Mutschke, H. 1997, ApJ, 476, 208

Bohren, C. F., \& Huffman, D. R. 1983, Absorption and Scattering of Light by Small Particles (New York: Wiley) 
Bordé, P., Coudé du Foresto, V., Chagnon, G., \& Perrin, G. 2002, A\&A, 393, 183

Cami, J., Yamamura, I., de Jong, T., et al. 2000, A\&A, 360, 562

Chagnon, G., Mennesson, B., Perrin, G., et al. 2002, AJ, 124, 2821

Cohen, M., Walker, R. G., Carter, B., et al. 1999, AJ, 117, 1864

Cotton, W. D., Mennesson, B., Diamond, P. J., et al. 2004, A\&A, 275, 288

David, P., \& Pégourié, B. 1995, A\&A, 293, 833

Drira, I., Huré, J. M., Spielfiedel, A., Feautrier, N., \& Roueff, E. 1997, A\&A, 319, 720

Gay, J., \& Mékarnia, D. 1988, in NOAO ESO Conf. on High Resolution Imaging by Interferometry, ed. F. Merkle, 811

Hale, D. D. S., Bester, M., Danchi, W. C., et al. 2000, ApJ, 537, 998

Kervella, P., Ségransan, D., \& Coudé du Foresto, V. 2004, A\&A, 425, 1161

Kholopov, P. N., Samus, N. N., Frolov, M. S., et al. 1988, General Catalogue of Variable Stars, 4th Edition, Vol. I-III

Knapp, G. R., Pourbaix, D., Platais, I., \& Jorissen, A. 2003, A\&A, 403, 993

Koike, C., Kaito, C., Yamamoto, T., et al. 1995, Icarus, 114, 203

Langhoff, S. R., \& Bauschlicher, C. W., Jr. 1993, Chem. Phys. Lett., 211, 305

Leinert, Ch., Graser, U., Przygodda, F., et al. 2003, Ap\&SS, 286, 73

Leinert, Ch., van Boekel, R., Waters, L. B. F. M., et al. 2004, A\&A, 423,537

Lorenz-Martins, S., \& Pompeia, L. 2000, MNRAS, 315, 856

Lovas, F. J., Maki, A. G., \& Olson, W. B. 1981, J. Mol. Spectros., 87, 449

Matsuura, M., Yamamura, I., Cami, J., Onaka, T., \& Murakami, H. 2002, A\&A, 383, 972

McCarthy, D. W., \& Low, F. J. 1975, ApJ, 202, L37
McCarthy, D. W., Low, F. J., \& Howell, R. 1977, ApJ, 214, L85

McCarthy, D. W., Howell, R., \& Low, F. J. 1978, ApJ, 223, L113 Mékarnia, D., \& Gay, J. 1990, A\&A, 238, 469

Mennesson, B., Perrin, G., Chagnon, G., et al. 2002, ApJ, 579, 446

Monnier, J. D., Geballe, T. R., \& Danchi, W. C. 1998, ApJ, 502, 833

Ohnaka, K. 2004a, A\&A, 421, 1149

Ohnaka, K. 2004b, A\&A, 424, 1011

Ohnaka, K., Balega, Y., Blöcker, T., et al. 2001, A\&A, 380, 212

Perrin, G., Coudé du Foresto, V., Ridgway, et al. 1999, A\&A, 345, 221

Perrin, G., Ridgway, S. T., Mennesson, B., et al. 2004a, A\&A, 426, 279

Perrin, G., Ridgway, S. T., Coudé du Foresto, V., et al. 2004b, A\&A, 418, 675

Rothman, L. S. 1997, HITEMP CD-ROM (Andover: ONTAR Co.)

Stecklum, B., MIDI Consortium, private communication, http://www . eso.org/observing/etc

Tipping, R. H., \& Chackerian, C., Jr. 1981, J. Mol. Spectros., 88, 352

Townes, C. H., Bester, M., Danchi, W. C., et al. 1998, Astronomical Interferometry, ed. R. D. Reasenberg, Proc. SPIE, 3350, 908

Tsuji, T., Ohnaka K., Hinkle, K. H., \& Ridgway, S. T. 1994, A\&A, 289, 469

Tsuji, T., Ohnaka K., Aoki, W., \& Yamamura, I. 1997, A\&A, 320, L1

Weiner, J., Danchi, W. C., Hale, D. D. S., et al. 2000, ApJ, 544, 1097

Weiner, J., Hale, D. D. S., \& Townes, C. H. 2003a, ApJ, 588, 1064

Weiner, J., Hale, D. D. S., \& Townes, C. H. 2003b, ApJ, 589, 976

Whitelock, P., Marang, F., \& Feast, M. 2000, MNRAS, 319, 728

Wittkowski, M., Aufdenberg, J., \& Kervella, P. 2004, A\&A, 413, 711

Wittkowski, M., Hummel, C. A., Johnston, K. J., et al. 2001, A\&A, 377,981

Woodruff, C., Eberhardt, M., Driebe, T., et al. 2004, A\&A, 421, 703

Yamamura, I., de Jong, T., \& Cami, J. 1999, A\&A, 348, L55 